\title{
Phase transformation accommodated plasticity in nanocrystalline nickel
}

\author{
X. Y. Zhang, ${ }^{1,4, a)}$ X. L. Wu, ${ }^{2, b)}$ Q. Liu, ${ }^{1}$ R. L. Zuo, ${ }^{1}$ A. W. Zhu, ${ }^{4}$ P. Jiang, ${ }^{2}$ and Q. M. Wei ${ }^{3}$ \\ ${ }^{1}$ School of Materials Sicence and Engineering, Chongqing University, \\ Chongqing 400030, People's Republic of China \\ ${ }^{2}$ State Key Laboratory of Nonlinear Mechanics, Institute of Mechanics, Chinese Academy of Sciences, \\ Beijing 100190, People's Republic of China \\ ${ }^{3}$ Department of Mechanical Engineering, University of North Carolina at Charlotte, Charlotte, \\ North Carolina 28223-0001, USA \\ ${ }^{4}$ Physical Science and Engineering Technology School, Guangxi University, \\ Nanning 530004, People's Republic of China
}

(Received 18 May 2008; accepted 12 June 2008; published online 21 July 2008)

\begin{abstract}
Based on detailed x-ray diffraction and transmission electron microscopy we have found body-centered-cubic (bcc) $\mathrm{Ni}$ upon room-temperature rolling of nanocrystalline (nc) face-centered-cubic (fcc) Ni. The bcc phase forms via the Kurdjumov-Sachs (KS) martensitic transformation mechanism when the von Mises equivalent strain exceeds $\sim 0.3$, much higher than accessible in tensile testing. The fcc and bcc phases keep either the KS or the NishiyamaWasserman orientation relationship. Our results provide insights into the deformation physics in nc $\mathrm{Ni}$, namely, the fcc-to-bcc phase transformation can also accommodate plasticity at large plastic strains. (C) 2008 American Institute of Physics. [DOI: 10.1063/1.2953545]
\end{abstract}

A good understanding of the deformation mechanisms of nanocrystalline (nc) materials is the key to microstructure design and property controls. ${ }^{1-6}$ Numerous efforts have corroborated that the grain boundary plasticity dominates via various partial dislocation activities during the plastic deformation of face-centered cubic (fcc) nc metals. ${ }^{1-10}$ It has been found, for example, that in $\mathrm{nc} \mathrm{Ni}$, deformation twinning, formation of extended, and full dislocations from the grain boundaries, grain boundary sliding, and grain rotation can contribute to plasticity. ${ }^{2,11-13}$ In this letter, we provide convincing $\mathrm{x}$-ray diffraction (XRD) and transmission electron microscopy (TEM) evidences for the formation of a bodycentered cubic (bcc, $\alpha$ ) phase of Ni via phase transformation in $\mathrm{nc}$ fcc $(\gamma) \mathrm{Ni}$ (grain size around $20 \mathrm{~nm}$ ) upon roomtemperature rolling. This observation is somewhat surprising as the $\gamma$ phase of Ni has been considered the exclusive equilibrium phase of nickel at ambient pressure and at temperatures up to its melting point, though both $\alpha$ and hexagonal close-packed phases of $\mathrm{Ni}$ have been synthesized mainly as very thin films by heteroepitaxial growth. ${ }^{14-17}$ On the other hand, molecular dynamics simulation reveals a continuous transformation even to an amorphous phase in $\mathrm{Ni}$ nanowires at a strain rate of $5 \% \mathrm{ps}^{-1}$, a stress of $9.5 \mathrm{GPa}$, and a strain of $15 \%$, respectively, with the stress level far exceeding the maximum flow stress of bulk Ni. ${ }^{18}$ The observations reported in this letter thus reveal yet another mechanism that can accommodate plasticity of $\mathrm{nc} \mathrm{Ni}$ at relatively large plastic strains.

In this work, fully dense, electrodeposited nc Ni sheets were procured from Goodfellow Inc. The as-received sheets are $200 \mu \mathrm{m}$ thick and of $99.8 \%$ purity. The average grain size is about $20 \mathrm{~nm}$. Samples of nc Ni of $10 \times 20 \mathrm{~mm}^{2}$ in size were rolled at room temperature to various von Mises equivalent strains, $\varepsilon_{\mathrm{VM}}$ Henceforth in this letter, $\varepsilon_{\mathrm{VM}}$ is calculated as $\varepsilon_{\mathrm{VM}}=|2 / \sqrt{3} \ln (1+\delta)|$, where $\delta$ is the rolling reduc-

\footnotetext{
a) Electronic mail: kehen888@163.com.

${ }^{b)}$ Electronic mail: xlwu@imech.ac.cn.
}

tion. The equivalent strain rate was approximately $10^{-2} \mathrm{~s}^{-1}$. The microstructures were examined using XRD and highresolution TEM (HRTEM, JEM 2010F operated at $200 \mathrm{kV}$ ). The TEM specimens were prepared by twin-jet polishing using a nitric acid methanol solution $\left(20 \%\right.$ of $\mathrm{HNO}_{3}$ by volume) at $-30{ }^{\circ} \mathrm{C}$.

Figure 1 displays the XRD results of nc Ni rolled to various equivalent strains. Only fcc peaks are found in the as-received nc $\mathrm{Ni}$ and it remains so to a $\varepsilon_{\mathrm{VM}}$ of around 0.27. However, when $\varepsilon_{\mathrm{VM}}$ is increased to around 0.32, extra XRD peaks, i.e., $(200)_{\alpha}$ and $(211)_{\alpha}$, start to appear which can be unequivocally indexed to be from the bcc phase of Ni. It therefore suggests that phase transition from fcc $(\gamma)$ to bcc $(\alpha)$ structure has occurred during rolling. Obviously, the formation of the $\alpha$ phase within the nc fcc matrix requires extensive plastic deformation with an equivalent strain far beyond what is accessible in a typical tensile test of nc $\mathrm{Ni}$ (usually below 5\%). ${ }^{19}$ The average lattice constants of $\gamma$ and

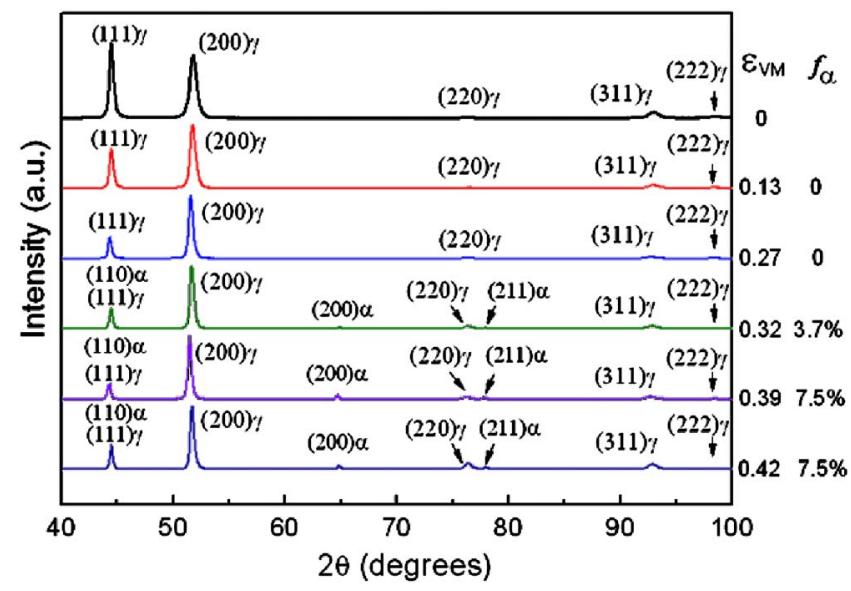

FIG. 1. (Color online) XRD results of as-received nc nickel and those rolled to various equivalent strains. Notice bcc peaks start to appear when the strain is around 0.32 . The strength of the bcc peaks increases with equivalent strain, implying increased volume fraction of the bcc phase. 

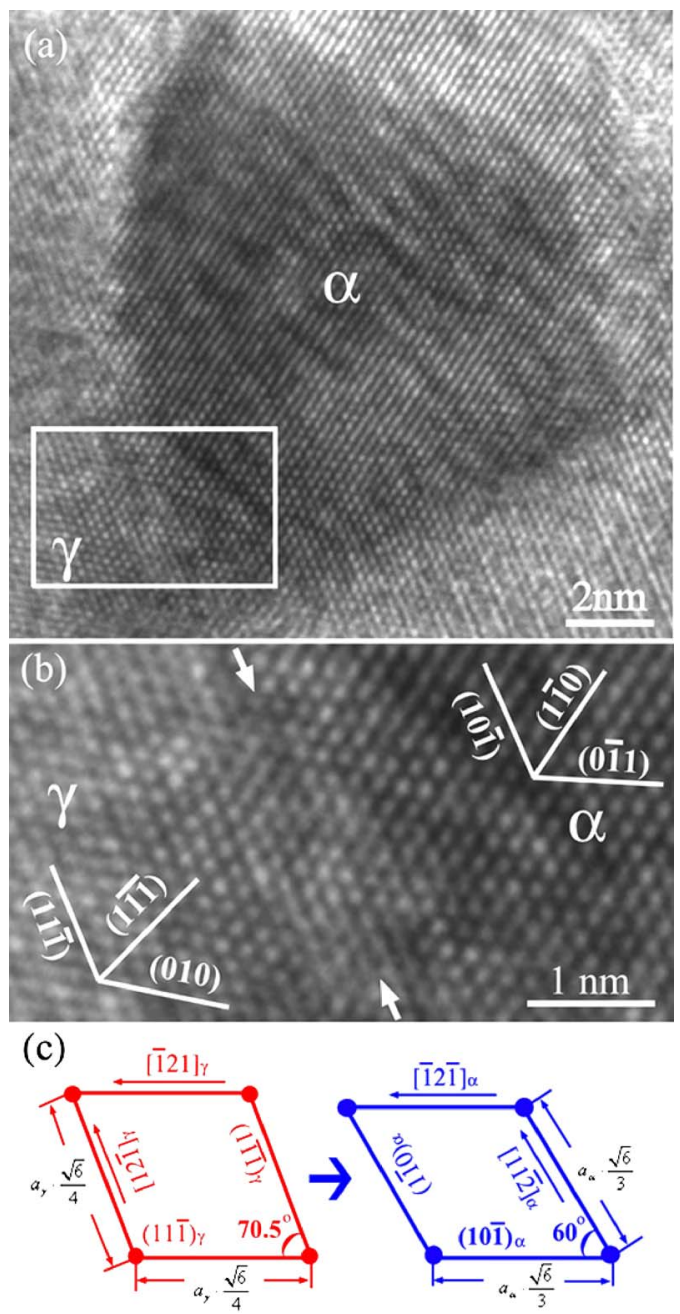

FIG. 2. (Color online) (a) HRTEM image of a nc bcc grain next to the fcc matrix. (b) Enlarged view of the interface region between the fcc and bcc structures. In (b), the atomic arrangements of the [101] zone of fcc and the [111] zone of bcc near the phase boundary are shown, with the $\{111\}$ $d$-spacing $(0.204 \mathrm{~nm})$ of the fcc phase and the $\{110\} d$-spacing $(0.206 \mathrm{~nm})$ of the bcc phase marked out, respectively. (c) Schematic configurations of the projected atomic arrays viewed in [101] of fcc and [111] of bcc.

$\alpha$ phase as measured by XRD are $a_{\gamma}=0.3532 \mathrm{~nm}$ and $a_{\alpha}$ $=0.2919 \mathrm{~nm}$, respectively. The latter is in good line with what is documented in the literature ${ }^{17,18}$ and is close to theoretical predictions. ${ }^{20}$ Further analysis of the XRD results indicates that the volume fraction of the $\alpha$ phase, $f_{\alpha}$, increases with equivalent strain, and reaches $7.5 \%$ at $\varepsilon_{\mathrm{VM}}$ of 0.43 .

The presence of $\alpha$ phase in the rolled nc $\mathrm{Ni}$ is further confirmed by HRTEM observation, as shown in Fig 2. Figure 2(a) shows a nc $\alpha$ grain viewed in the $[111]_{\alpha}$ zone axis of the bcc lattice. Figure 2(b) provides an enlarged view of the boxed area in Fig. 2(a) containing the $\gamma / \alpha$ phase boundary, as indicated by arrows. The zone axes of the $\gamma$ and $\alpha$ structures are $[101]_{\gamma}$ and $[111]_{\alpha}$, respectively. It is evident that $(11 \overline{1})_{\gamma}$ is parallel to $(10 \overline{1})_{\alpha}$, in accordance with the Kurdjumov-Sachs (KS) orientation relationship well established for the $\gamma$-to- $\alpha$ martensitic transformation in low carbon ferrous alloys. ${ }^{21,22}$ The schematic projected configurations of atomic arrays, viewed in the respective zone axes of the $\gamma$ and $\alpha$ phases, are shown in Fig. 2(c). The observed and calibrated spacings between these atomic rows on $\{111\}_{\gamma}$ and $\{110\}_{\alpha}$ planes are both $0.22 \mathrm{~nm}$. This is in good keeping with Downloaded 30 Jul 2009 to 159.226.231.70. Redistribution subject

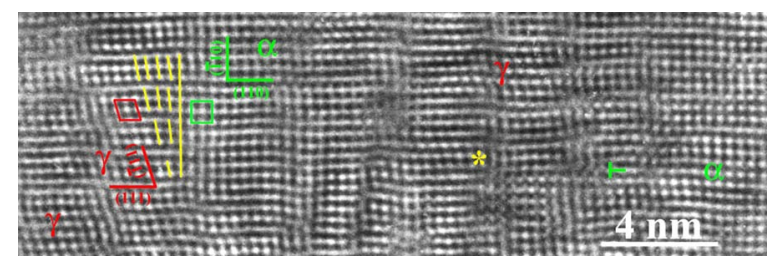

FIG. 3. (Color online) HRTEM image showing coexistence of the fcc (diamond) and bcc (square) phases in a nc nickel after room-temperature rolling, and the transitional structures indicated by an asterisk between the original fcc structure and the final bcc structure.

the calculated values of $0.2156 \mathrm{~nm}\left(a_{\gamma} \sqrt{6} / 4\right)$ and $0.2383 \mathrm{~nm}$ $\left(a_{\alpha} \sqrt{6} / 3\right)$ for the $\gamma$ and $\alpha$ phases, respectively. It is seen from Fig. 2(c) that when the lattice is transformed completely from $\gamma$ to $\alpha$, the resultant amount of shear is $(\sqrt{6} / 24) /(\sqrt{3} / 3)=\sqrt{2} / 8=0.177$, as obtained from the geometry of the projected atomic rows shown in Fig. 2(c). In other words, this shear is produced by the displacement of each $(11 \overline{1})_{\gamma}$ plane in the $[\overline{1} 21]_{\gamma}$ direction by $a_{\gamma} \sqrt{6} / 24$. The equivalent strain corresponding to the lattice change described by Fig. 2(c) is $\sim 0.231$.

One important feature is noted, from Figs. 2(b) and 2(c), that the $(111)_{\gamma}$ plane is gradually curved into $(110)_{\alpha}$ plane, indicating the presence of various stages of transient structures. An example is shown in Fig. 3. In this HRTEM image, the diamond and the square lattices correspond to the $\gamma$ and $\alpha$ phases as viewed in zone axes of $[01 \overline{1}]_{\gamma}$ and $[00 \overline{1}]_{\alpha}$, respectively. Clearly, the Nishiyama-Wasserman orientation relationship, viz., $[00 \overline{1}]_{\alpha} \|[01 \overline{1}]_{\gamma}$ and $(110)_{\alpha} \|(111)_{\gamma}$ can be established, which is also a typical orientation relationship found in martensitic transition in ferrous alloys. ${ }^{21-23}$ This result again provides strong evidence for the occurrence of $\gamma$ $\rightarrow \alpha$ martensitic transformation in nc Ni. Moreover, it is worth noting that the $\gamma \rightarrow \alpha$ transformation take places via a continuous shearing process, by the formation of an intermediate structure between the final bcc and the original fcc structure, and one typical area is marked by an asterisk in Fig. 3. The intermediate structures have their orientations deviating away from $[01 \overline{1}]_{\gamma}$. This continuous shearing process is also supported as a number of dislocations (indicated by short yellow lines in Fig. 3) is visible which stacks up at the boundary between the $(\overline{111})_{\gamma}$ and $(\overline{1} 10)_{\alpha}$ planes. These dislocations help to provide the gradual tilting from the $(111)_{\gamma}$ to the $(\overline{1} 10)_{\alpha}$ planes, which eventually leads to the formation of the bcc $(\alpha)$ phase. Interestingly, one dislocation is also observed in $\alpha$ phase, as indicated by symbol $\mathrm{T}$ in Fig. 3.

Crystallographically, when the KS orientation relationship is described as $(11 \overline{1})_{\gamma} \|(10 \overline{1})_{\alpha}$ and $[101]_{\gamma} \|[111]_{\alpha}$, as adopted in Fig. 2, the first and second shears in the KS mechanism are $[\overline{2} 1 \overline{1}]_{\gamma}(11 \overline{1})_{\gamma}$ and $[101]_{\gamma}(1 \overline{21})_{\gamma}$, respectively. The amounts of shears are 0.354 for the first shear and 0.199 for the second shear, which are produced by a shear displacement of $a_{\gamma} / 12 \cdot[\overline{2} 1 \overline{1}]_{\gamma}$ on every $(11 \overline{1})_{\gamma}$ plane and a shear displacement $a_{\gamma} / 12 \cdot[101]_{\gamma}$ on every third $(\overline{2} 42)_{\gamma}$ plane. $^{23,24}$ It is noted that in the KS mechanism, the shear component of the first shear along $[\overline{1} 21]_{\gamma}$ on the $(11 \overline{1})_{\gamma}$ plane is 0.177 , which is exactly the same as the observed amount of the apparent shear in $[\overline{1} 21]_{\gamma}$ on the $(11 \overline{1})_{\gamma}$ plane, deduced from the atomic arrangements as projected to the plane normal to the incident beam [Fig. 2(c)]. Moreover, in the KS mechao AIP license or copyright; see http://apl.aip.org/apl/copyright.jsp 
nism, the direction of the second shear is parallel to the incident beam, so that the atomic displacement along this direction does not affect the image formation of the atomic structure in the electron microscope. As a consequence, the positions of atomic rows projected along this incident beam are expected to remain unchanged even if the $\gamma$ phase is subjected to the second shear. In other words, if the KS mechanism is operating, the HRTEM image along $[101]_{\gamma}$ and $[111]_{\alpha}$ will remain unchanged. The relatively undisturbed image of the atomic rows corresponding to the fcc and bcc structures in Fig. 2(b) strongly supports the above supposition. Therefore, the KS mechanism is the plausible lattice deformation in the $\gamma \rightarrow \alpha$ martensitic transformation that happened in nc Ni.

Thermodynamically, theoretical calculations have suggested that the hypothetical bcc phase of Ni can be formed by transition from the fcc phase at an energy cost of $6.68 \mathrm{~kJ} / \mathrm{mol} .{ }^{20}$ This energy cost must be provided from mechanical driving force. We envisage that the very high yield strength of $\mathrm{nc} \mathrm{Ni}$ and the stress condition of rolling together may serve to provide such energy cost. A simplified calculation of the mechanical work accumulated during rolling of nc $\mathrm{Ni}$ can be made by assuming that the stress-strain behavior follows elastic-perfect plastic pattern, and the strain energy is then the area under the stress-strain curve. Such estimate reaches an overall volume average value of $\sim 4 \mathrm{~kJ} / \mathrm{mol}$ at an equivalent strain of 0.32 . Therefore, a localized phase change from fcc-to-bcc structure should not be very surprising from a thermodynamic point of view.

The $\alpha$ phase of nc Ni formed during rolling can be, in fact, referred to as a mechanically induced martensite transformation. We observed nc grains of either a whole bcc lattice (Fig. 2) or a transient one (Fig. 3). When the nc Ni is subjected to an external force, an inhomogeneous state of internal stress is developed resulting from elastic anisotropy and plastic incompatibilities in different grains or even within different parts of a grain. ${ }^{6,12}$ The occurrence of transformation is, as expected, dependent on deformation conditions. In our case, it depends on the corresponding very high flow stress and appropriate strain levels. The accommodation for compatible plastic strains is thus nonuniform, demonstrating the inhomogeneous nature of the deformation accommodation. ${ }^{12}$ More specifically, the plastic deformation may also vary from place to place even in one single grain. There appear to be multiple different deformation responses that can accommodate plastic strain even in one single nc grain. We also observed deformation twining and formation of stacking faults and dislocations in rolled nc grains, showing that strain accommodation has multiple pathways and is inhomogeneous from place to place in the nc grain. Our results extend the prior knowledge of plastic accommodation in nc metals with convincing evidence of microstructural in- stability via phase transformation occurring in $\mathrm{nc} \mathrm{Ni}$ at relatively large strains.

In summary, we have provided strong XRD and HRTEM evidence for the formation of a bcc structure in $\mathrm{nc}$ fcc $\mathrm{Ni}$ upon room-temperature rolling to large strains. The formation of the bcc phase is attributed to a mechanically driven martensitic phase transformation that occurs within nc nickel. The lattice instability observed here is of considerable practical importance. The "hard-to-flow" far-fromequilibrium microstructure such as nc Ni can accommodate plastic strain via change in lattice structure into another variant upon mechanical loading. This mechanism adds to the host of plastic deformation mechanisms of nc nickel.

This work was supported by NSFC, Grant Nos. 50571110, 10721202 and 50461001, CAS Grant No. KJCX2-YW-M04, and MOST Grant No. 2004CB619305.

${ }^{1}$ V. Yamakov, D. Wolf, S. R. Phillpot, A. Mukherjee, and H. Gleiter, Nat. Mater. 1, 1 (2002); 3, 43 (2004).

${ }^{2}$ H. Van Swygenhoven, P. M. Derlet, and A. Froseth, Nat. Mater. 3, 399 (2004).

${ }^{3}$ M. W. Chen, E. Ma, K. J. Hemker, H. W. Sheng, Y. M. Wang, and X. M. Cheng, Science 300, 1275 (2003).

${ }^{4}$ R. J. Asaro, P. Krysl, and B. Kad, Philos. Mag. 83, 733 (2003).

${ }^{5}$ M. A. Meyers, A. Mishra, and D. J. Benson, Prog. Mater. Sci. 51, 427 (2006).

${ }^{6}$ Y. T. Zhu, X. Z. Liao, S. G. Srinivasan, Y. H. Zhao, M. I. Baskes, F. Zhou, and E. J. Lavernia, Appl. Phys. Lett. 85, 5049 (2004).

${ }^{7}$ X. Z. Liao, F. Zhou, E. J. Lavernia, S. G. Srinivasan, I. Baskes, D. W. He, and Y. T. Zhu, Appl. Phys. Lett. 83, 632 (2003).

${ }^{8}$ X. Z. Liao, F. Zhou, E. J. Lavernia, D. W. He, and Y. T. Zhu, Appl. Phys. Lett. 83, 5062 (2003).

${ }^{9}$ J. Weissmuller and J. Markmann, Adv. Eng. Mater. 7, 202 (2005).

${ }^{10}$ J. Wang and H. C. Huang, Appl. Phys. Lett. 85, 5983 (2004).

${ }^{11}$ Z. W. Shan, E. A. Stach, J. M. K. Wiezorek, J. A. Knapp, D. M. Follstaedt, and S. X. Mao, Science 305, 654 (2004).

${ }^{12}$ X. L. Wu and E. Ma, Appl. Phys. Lett. 88, 061905 (2006); 88, 231911 (2006); J. Mater. Res. 22, 2241 (2007).

${ }^{13}$ X. Wu, Y. T. Zhu, M. W. Chen, and E. Ma, Scr. Mater. 54, 1685 (2006).

${ }^{14}$ C. S. Tian, D. Qian, D. Wu, R. H. He, Y. Z. Wu, W. X. Tang, L. F. Yin, Y. S. Shi, G. S. Dong, X. F. Jin, X. M. Jiang, F. Q. Liu, H. J. Qian, K. Sun, L. M. Wang, G. Rossi, Z. Q. Qiu, and J. Shi, Phys. Rev. Lett. 94, 137210 (2005).

${ }^{15}$ W. Tian, H. P. Sun, X. Q. Pan, J. H. Yu, M. Yeadon, C. B. Boothroyd, Y. P. Feng, R. A. Lukaszew, and R. Clarke, Appl. Phys. Lett. 86, 131915 (2005).

${ }^{16}$ J. G. Wright and J. Goddard, Philos. Mag. 11, 485 (1965).

${ }^{17}$ J. Narayan, J. Chen, R. M. Moon, and R. W. Carpenter, Philos. Mag. A 49, 287 (1984).

${ }^{18}$ H. Ikeda, Y. Qi, T. Cagin, K. Samwer, W. L. Johnson, and W. A. Goddard, Phys. Rev. Lett. 82, 2900 (1999).

${ }^{19}$ Y. M. Wang, A. V. Hamza, and E. Ma, Appl. Phys. Lett. 85, 2750 (2004).

${ }^{20}$ K. P. Thakur and S. Ahmad, Phys. Status Solidi B 144, 529 (1987).

${ }^{21}$ J. W. Christian and D. V. Edmonds, in Phase Transformations in Ferrous Alloys, edited by A. R. Marder and J. L. Goldstein (ASM, Metals Park, OH, 1984), pp. 293-325.

${ }^{22}$ G. B. Olsen and M. Cohen, Acta Metall. 27, 1907 (1979).

${ }^{23}$ S. Kajiwara, K. Ogawa, and T. Kikuchi, Philos. Mag. Lett. 74, 405 (1996).

${ }^{24}$ J. W. Christian, Metall. Trans. A 21A, 799 (1990). 